\title{
Exploring Barrier Mechanisms Relevant for Drug Therapy
}

\author{
Heidi Wunderli-Allenspach*, Karsten Bucher, Jiri Hofmann, Marco Marenchino, Anita Thomae, \\ Maja Günthert, and Stefanie D. Krämer
}

\begin{abstract}
Membranes play an important role in the compartmentalization of cells and organs. Up to 500 different lipids have been reported to be present in different biological membranes. The origin and meaning of this diversity is not well understood. Membranes mediate the necessary separation between organizational units, but also provide the possibility to connect them. Overcoming the membrane barriers within the body is a major task for any therapeutic agent. The research of the Wunderli group is concentrated on elucidating the basis of drug-membrane interaction and permeation processes, on unraveling the mechanism of action of multi-substrate membrane transporters such as the multi-drug resistance protein P-glycoprotein (P-gp), and on the modulation of paracellular diffusion. Three major projects are pursued: (1) drug-membrane interaction and permeation studies with liposomes of different lipid composition; (2) studies on the mechanism of action of the P-gp with proteoliposomes; (3) modulation of the tight junctions (TJs) of cell barriers to enhance the paracellular transport of hydrophilic therapeutic entities, e.g. peptides.
\end{abstract}

Keywords: Lipid membranes · Multi-drug resistance $\cdot$ Partitioning $\cdot$ Permeation $\cdot$ Tight junctions

\section{Introduction}

Looking at the pharmacokinetics of drugs (ADME: absorption, distribution, metabolism, excretion), the crossing of barriers is a major theme in drug therapy (Fig. 1). For instance, drugs have to pass the mucosa of the gastrointestinal tract or penetrate through the skin before being distributed in the body via the circulation system. Even if a molecule is brought directly into the blood stream by injection, it has to permeate through the lining of the capillary vessels (endothelia) in order to reach its target. For the biotransformation of drugs the crossing of cell membranes is crucial to reach the metabolizing enzymes. Finally, the re-absorption of drug from the urine back into the blood through the renal epithelia is a major determinant for the net clearance of a therapeutic agent.

${ }^{*}$ Correspondence: Prof. Dr. H. Wunderli-Allenspach Department of Chemistry and Applied Biosciences Swiss Federal Institute of Technology

ETH Hönggerberg

$\mathrm{CH}-8093$ Zürich

Tel. +41446337431

E-Mail: heidi.wunderli@pharma.ethz.ch

http://www.pharma.ethz.ch
Today, drug development is still hampered by a large attrition rate. Nearly $40 \%$ of the failures are related to pharmacokinetic problems. Therefore, it is mandatory that assays with a better predictive value be established for pharmacokinetic profil- ing at an early stage in development. This is only possible if a better understanding of basic mechanisms in drug-membrane interaction, drug permeation through membranes, carrier-mediated transport and the subtle modulation of TJs is achieved.

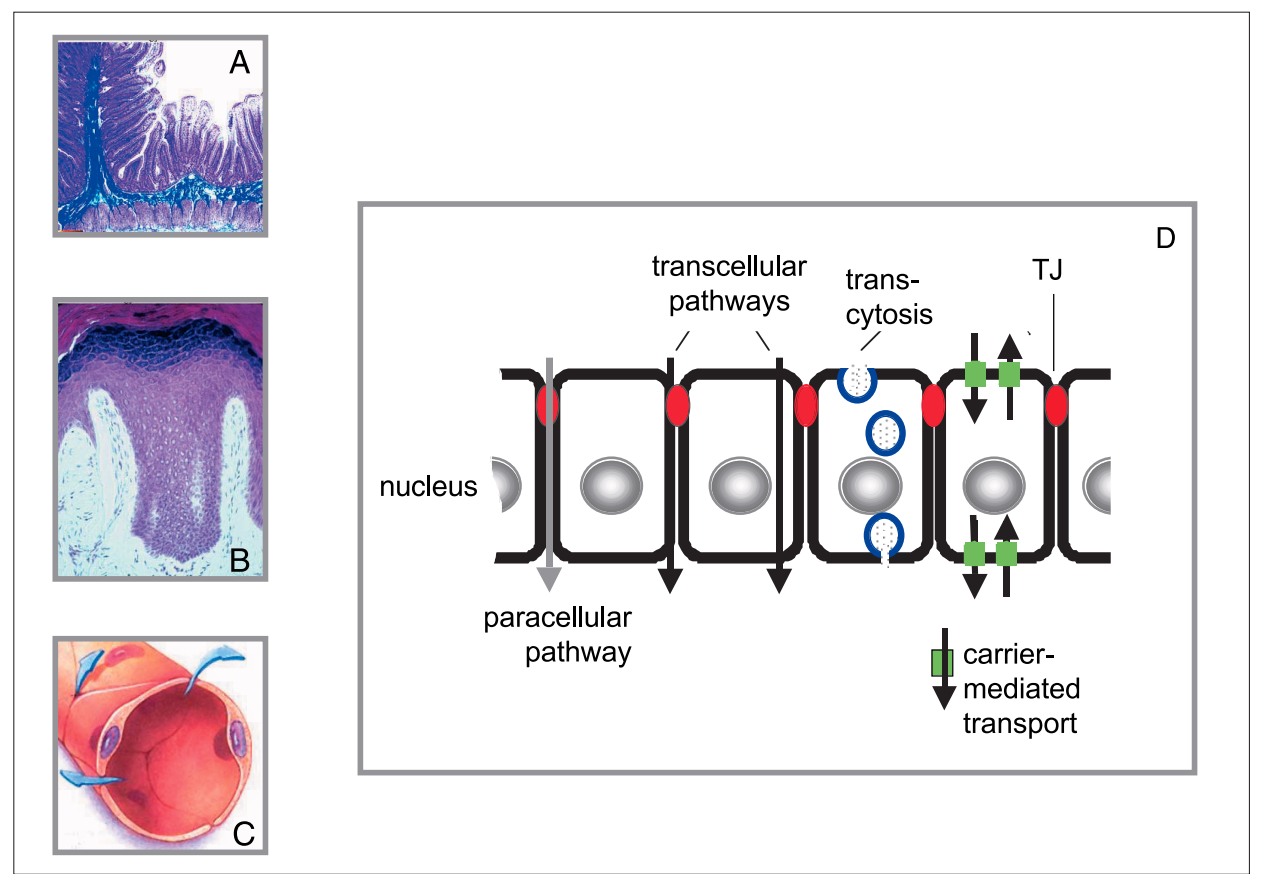

Fig. 1. Cellular barriers in man. (A) Intestinal wall, total surface $\sim 200 \mathrm{~m}^{2}$; (B) skin, total surface 1.5-2 $\mathrm{m}^{2}$; (C) capillary blood vessel, total surface $\sim 300 \mathrm{~m}^{2}$. (D) Schematic representation of drug permeation through cellular barriers (epithelia, endothelia) by different routes. 


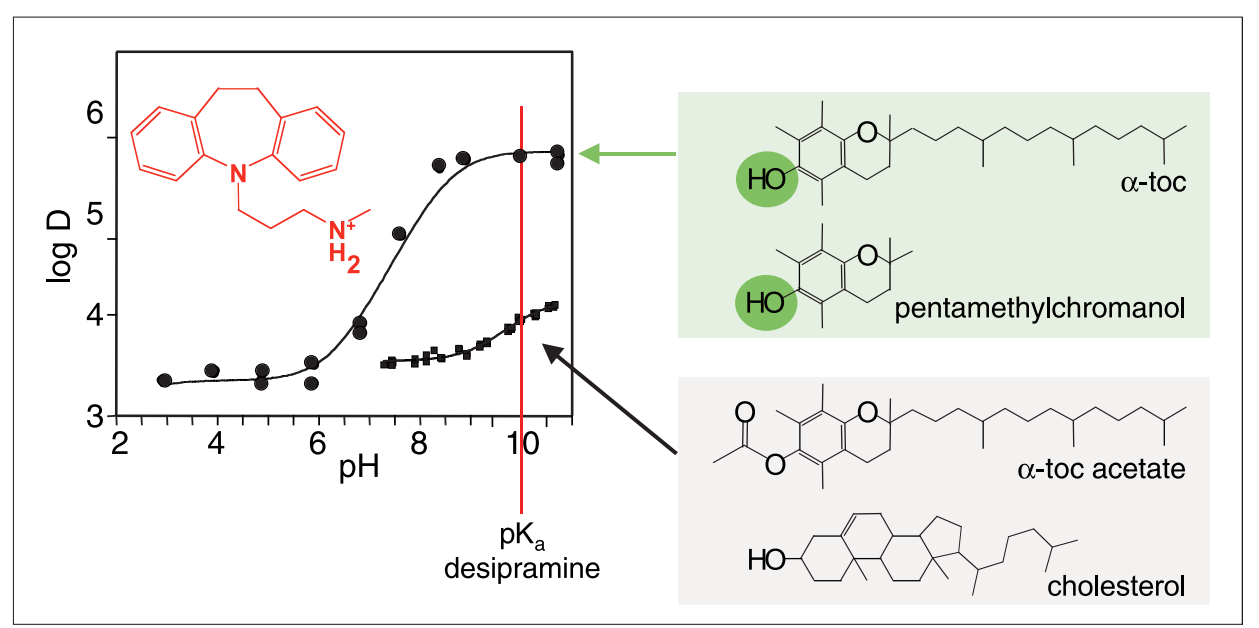

Fig. 2. Influence of membrane composition on the $\mathrm{pH}$-distribution profile of desipramine in liposomes. Liposomes were produced with different composition as indicated, and equilibrium dialysis was performed in the presence of ${ }^{3} \mathrm{H}$-desipramine at $37^{\circ} \mathrm{C}$ at $\mathrm{pH}$ values between 3 and 10.5. The concentrations in both chambers were determined by liquid scintillation counting and the distribution coefficients $(\log \mathrm{D})$ were calculated as previously described [1]. (•) type I partitioning: liposomes with PC and 20\% (mol/mol) alpha-tocopherol, and PC and pentamethylchromanol, respectively; (匚) type II partitioning: liposomes with PC only, PC and alpha-tocopherolacetate, and PC and cholesterol, respectively.

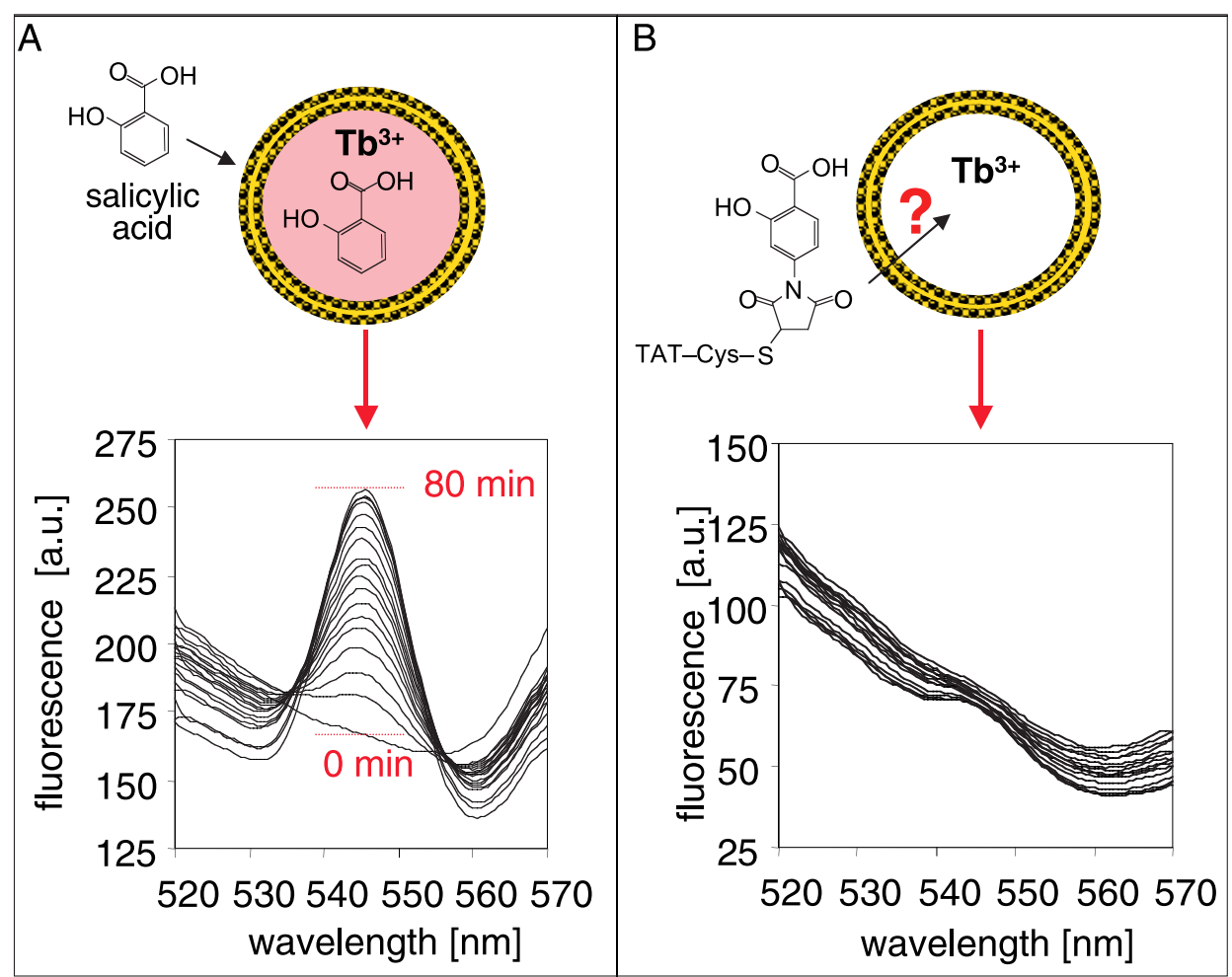

Fig. 3. Liposomal $\mathrm{Tb}^{3+}$ assay to study the permeation of aromatic carboxylic acids through lipid bilayers. The proof of principle is shown with the permeation of salicylic acid through the membrane of $\mathrm{Tb}^{3+}$-containing PC liposomes (A). The increase of fluorescence upon the interaction of permeated salicylic acid with $\mathrm{Tb}^{3+}$ molecules is followed during $80 \mathrm{~min}$ (for details see [9]). No significant increase in fluorescence was detected upon incubation with the cell penetrating peptide TAT (44-57)-Cys linked to 4-maleimidosalicylic acid (B).

\section{Drug-Membrane Interaction and Permeation Studies}

To study drug-membrane affinity with membranes of different lipid composition, a liposomal partitioning system using equilibrium dialysis has been established [1-3].
As could be shown (Fig. 2), the extent of drug partitioning varies significantly with the composition of the lipid bilayer. For instance, a hundred-fold increase in the affinity of neutral desipramine is found in alphatocopherol- or pentamethylchromanol-containing phosphatidylcholine (PC) lipo- somes as compared to PC liposomes only. In contrast, this increase in affinity is not found if alpha-tocopherolacetate or cholesterol is present in PC liposomes. This indicates that the membrane affinity of a drug is strikingly dependent on the lipid composition of the bilayer, and that even the presence of minor lipids, such as $1.5 \%$ alpha-tocopherol, are decisive for partitioning [4]. Increased membrane affinity for protonated basic drugs is found if phosphatidylinositol or fatty acids (FFA) are included in the membranes [5-7]. Slight changes in the environmental $\mathrm{pH}$ of FFA-containing membranes can lead to significant differences in the affinity for drug molecules due to the $\mathrm{pH}$-sensitive extent of ionization of FFA around $\mathrm{pH}$ 7. The therapeutic relevance of this finding has not been explored yet. In the special case of drug-induced lipid aggregation in lysosomes ( $\mathrm{pH} \sim 4.5$ ), so-called phospholipidosis, it is known that the effect can be reversed by addition of alpha-tocopherol to cell cultures and in vivo [8]. Our findings suggest that redistribution of the drug to non-lyposomal membranes due to an increase in the membrane affinity at $\mathrm{pH} 7$ in the presence of alpha-tocopherol is the underlying mechanism of the reversion.

More recently we have extended our affinity studies to the arrangement of endogenous peptides in membrane domains. This project relies on NMR studies and is pursued in collaboration with Beat Meier (ETH Zürich).

Permeation of drugs through membranes is still poorly understood. A new liposomal assay based on the reaction of aromatic carboxylic acids with terbium ${ }^{3+}$ $\left(\mathrm{Tb}^{3+}\right)$ has been developed in our group to study permeation through lipid membranes [9]. The $\mathrm{Tb}^{3+}$ is included in the aqueous lumen of liposomes where it reacts with incoming carboxylic acids that permeate through the lipid membrane. Permeation can be monitored as an increase of fluorescence over time. The proof of principle for the $\mathrm{Tb}^{3+}$ assay was made with salicylic acid that penetrates through PC liposomes (Fig. $3 \mathrm{~A})$. It could be shown with this assay that the membrane-permeating characteristics reported for a sequence of the HIV TAT protein are not based on passive permeation but may rather be related to endocytosis (Fig. 3B). The $\mathrm{Tb}^{3+}$ assay, if necessary combined with stopped flow technology, is a powerful tool to study the permeation kinetics of different aromatic carboxylic acids thus giving a clue to the little understood mechanism of molecule permeation through lipid bilayers of different composition. Systematic variation of the lipid composition of liposomes should shed new light on the permeation of different molecules through cell membrane domains. 


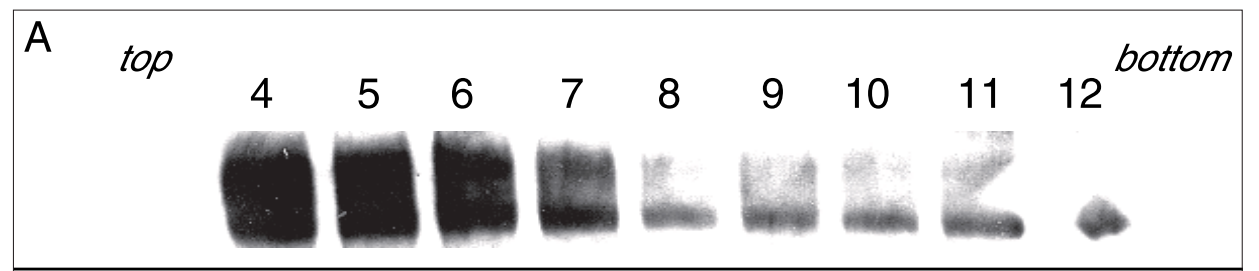

B

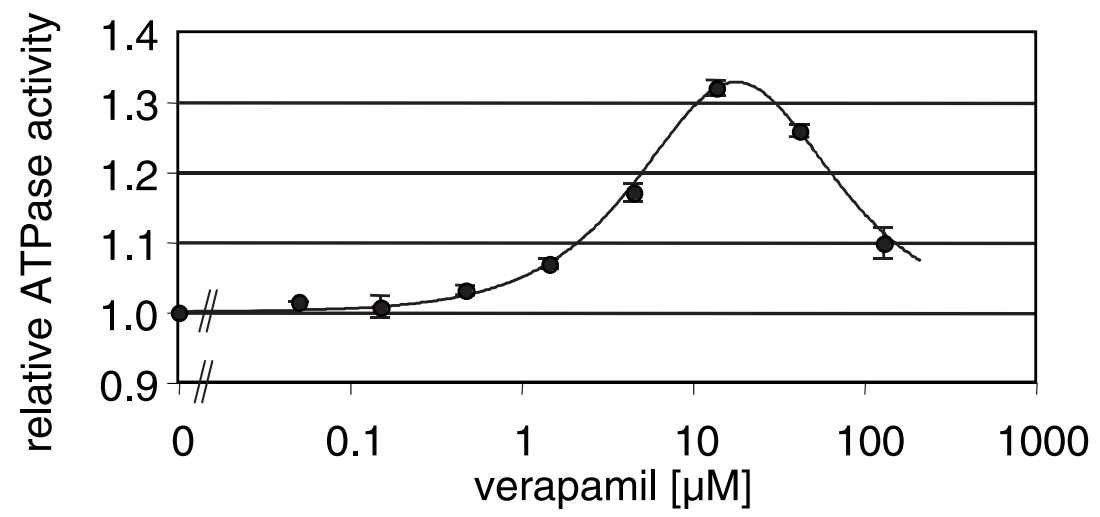

Fig. 4. Hydrolysis of ATP as a functional assay for P-gp. Low temperature detergent-resistant membrane domains (rafts) were isolated from P388/ARD cells and purified by sucrose gradient density centrifugation. (A) Western blot analysis of different gradient fractions with the anti human P-gp antibody C219. (B) Effect of verapamil, a P-gp ATPase activating compound, on the ATPase activity in rafts. Data were fitted based on the Michaelis Menten equation assuming inhibition by high substrate concentrations.

\section{P-gp, a Multi-substrate Trans- porter Glycoprotein}

Beside passive permeation, carrier-mediated processes play an important role in drug distribution and elimination. One of the transporters, the P-gp, a $180 \mathrm{kDa}$ membrane protein, has been identified as one of the major causes for chemotherapy resistance of a wide variety of tumors. This socalled multi-drug resistance protein has been sequenced and the possible transmembrane domains of the protein have been designated. The mechanism of action remains unclear and controversial hypotheses exist. Screening studies identified a number of clinically important drugs as P-gp substrates, which are as diverse as anthracyclines, alkaloids, peptides, steroid hormones, and local anesthetics, furthermore pharmaceutical excipients and dye molecules such as rhodamine 123 . The latter can be used in an efflux assay for the screening of P-gp inhibitors in the confocal laser scanning microscope (CLSM) [10]. The multi-substrate recognition of $\mathrm{P}$-gp hints at unusual characteristics as compared to classical carriers such as peptide transporters. ATP binding and hydrolysis is essential for the transport function of P-gp. ATPase activity can thus be used as a functional assay to study P-gp activity with different substrates and inhibitors. An easy to handle assay for P-gp activity has recently been introduced (Bucher et al., manuscript in preparation). It is based on the use of a P-gp preparation obtained by low temperature detergent extraction of the glycoprotein from adriamycin-resistant murine lymphoma cells (P388/ADR). The resulting socalled raft fractions are highly enriched in P-gp and can be used directly to measure ATP hydrolysis. This experimental set-up can be used to establish ATPase activity patterns for typical substrates, inhibitors and modulators (Fig. 4).

To study the influence of defined lipid surroundings on the activity of the multidrug resistance protein, which is also postulated to be a lipid-sorting protein, a procedure is being developed in our lab to purify the P-gp and integrate it into proteoliposomes. This is to explore potential lipid substrates and to check whether the interactions of drugs with the lipid surroundings of the protein are involved in substrate recognition. Recent progress has been made to produce unilamellar vesicles with $\mathrm{P}-\mathrm{gp}$ as the major protein and negligible amounts of residual detergent. Proteoliponow be used to study changes in the ATP hydrolysis patterns of substrates and inhibitors previously characterized with raft fractions.

\section{Modulation of TJs to Enhance Permeation Through Cellular Barriers}

Tight junctions (TJs) separate distinct components of the apical and basolateral surface domains of polarized cells and at somes of different lipid composition will the same time act as a dynamic gate to regulate passage of molecules through the paracellular pathway. Understanding TJ regulation processes can significantly help to improve drug delivery through the epithelial or brain endothelial barrier while preventing cell damage. The reversible opening of TJs can be demonstrated using CLSM with a fusion construct (chimera) between green fluorescent protein (GFP) and ZO-1, a major TJ protein, stably expressed in Madin Darby Canine kidney (MDCK) cells (Fig. 5; [11]). When using the transient opening of TJs for drug permeation enhancement, mild conditions have to be applied that avoid inflammatory reactions at the site of application. Interesting candidates for a controlled partial opening of TJs are peptides corresponding to the
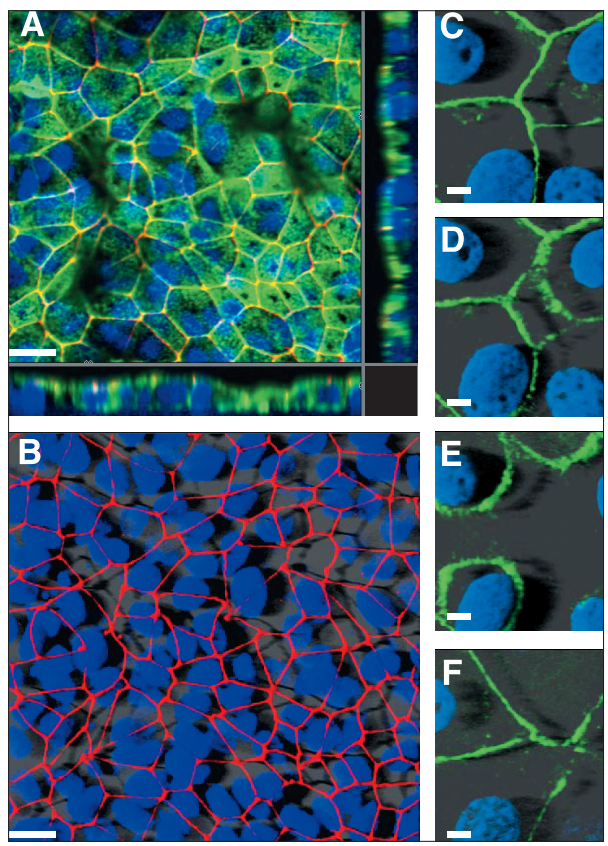

Fig. 5. The TJ network in Madin Darby Canine Kidney (MDCK) cells and the reversible opening of TJs as visualized with the GFP-ZO1 protein in the CLSM. Cells were grown under standard conditions and prepared for CLSM as previously described [11]. (A) Optical section through fixed MDCK cell monolayer $(x, y)$ labeled for nuclei (blue), F-actin (green) and the TJ protein ZO-1 (red). Co-localization of F-actin and ZO-1 appears as yellow staining. The cross section through the monolayer can be seen in the $x, z-$ and y,z-projections. (B) Same area as in (A) in the 'shadow projection' mode, showing only nuclei (blue) and the TJ network by labeling ZO-1 (red). (C) to (F) Time lapse sequence taken in living transfected MDCK cells expressing the green fluorescent protein (GFP)-ZO1 fusion protein (ZO1CGFP-MDCK) treated with 2 mM EGTA. Optical section through the $x, y$ level: GFP-ZO1 (green), nuclei (blue) labeled with a life stain. (C) control at 0 min; (D) 7 min EGTA; (E) 16 min EGTA; (F) 16 min in EGTA plus 70 min in normal, $\mathrm{Ca}^{2+}$-containing culture medium. Bars in $A$ and $B$ represent $10 \mu \mathrm{m}$; bars in $\mathrm{C}$ to $\mathrm{F}$ represent $5 \mu \mathrm{m}$. 


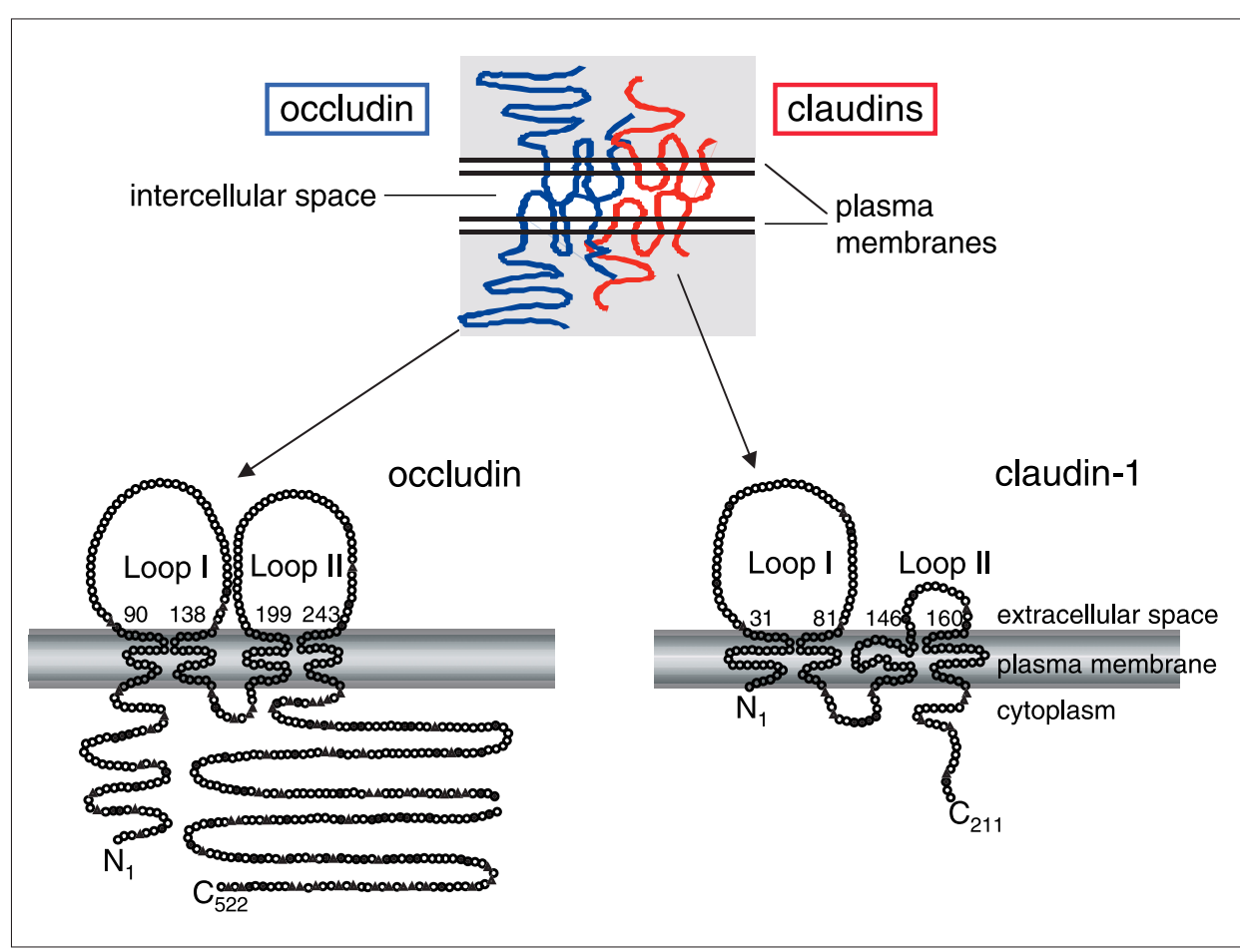

Fig. 6. Hypothetical arrangement of the transmembrane TJ proteins occludin and claudin. The loop regions contain stretches of hydrophobic amino acids that are assumed to contribute to the tightness of the TJs by making contacts between adjacent cells.

extracellular loops of occludin and claudin isoforms, two types of transmembrane TJ proteins (Fig. 6). The project concentrates on the production of peptides with TJ modulating potential that could be used for the development of permeation enhancers. Opening of the TJs can be quantified by determining the transepithelial electrical resistance, and by measuring mannitol transport in a two-chamber cell culture system. Preliminary data show some effect of peptides corresponding to the occludin loop II and the claudin-1 loop I (Fig. 7).

Received: July 21, 2004

[1] G.M. Pauletti, H. Wunderli-Allenspach, Eur. J. Pharm. Sci. 1994, 1, 27.

[2] S.D. Krämer, H. Wunderli-Allenspach, Pharm. Res. 1996, 13, 1850.

[3] C. Ottiger, H. Wunderli-Allenspach, Eur. J. Pharm. Sci. 1996, 5, 223.

[4] M. Marenchino, A.L. Alpstäg-Wöhrle, B. Christen, H. Wunderli-Allenspach, S.D. Krämer, Eur. J. Pharm. Sci. 2004, 21, 313.

[5] S.D. Krämer, A. Braun, C. Jakits-Deiser, H. Wunderli-Allenspach, Pharm. Res. 1998, 15, 739.

[6] S.D. Krämer, C. Jakits-Deiser, H. Wunderli-Allenspach, Pharm. Res. 1997, 14, 827.

[7] S.D. Krämer, in 'Pharmacokinetic Optimization in Drug Research: Biological, Physicochemical and Computational Strategies', Eds. B. Testa, H. van de Waterbeemd, G. Folkers, R. Guy. WileyVCH, Weinheim, 2001, p. 401.
[8] U.E. Honegger, L. Scuntaro, U.N. Wiesmann, Biochem. Pharmacol. 1995, 49, 1741.

[9] S.D. Krämer, H. Wunderli-Allenspach, Biochim. Biophys. Acta-Biomembr. 2002, 1609, 161.

[10] S.P. Hämmerle, B. Rothen-Rutishauser, S.D. Krämer, M. Günthert, H. WunderliAllenspach, Eur. J. Pharm. Sci. 2000, 12, 69.

[11] F.K. Riesen, B. Rothen-Rutishauser, H. Wunderli-Allenspach, Histochem. Cell Biol. 2002, 117, 307.

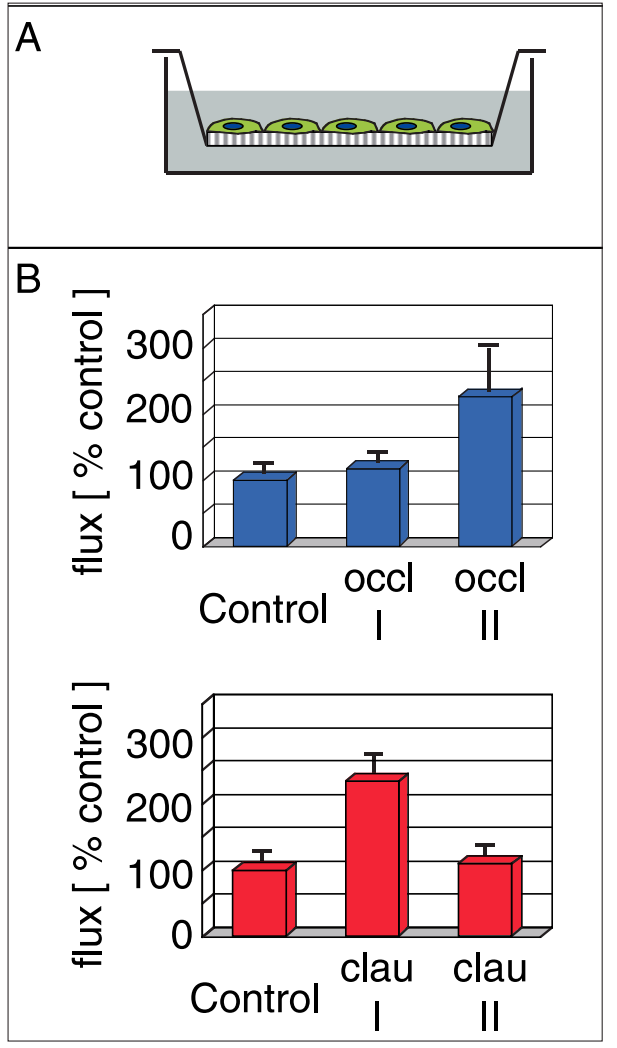

Fig. 7. The mannitol permeation assay to test the opening of TJs. Cells were grown to confluence on membrane inserts and incubated in a twochamber system (A). ${ }^{3} \mathrm{H}$-mannitol was added to the upper chamber in the presence or absence of the indicated peptides, and samples were taken from the lower chamber at different times. Data are presented for a $48 \mathrm{~h}$ incubation period. Partial opening of TJs is reflected in an increase in the permeation of ${ }^{3} \mathrm{H}$-mannitol expressed as flux in percentage of the control without peptides. Increased permeation of ${ }^{3} \mathrm{H}$-mannitol is paralleled by a decrease in the transepithelial electrical resistance (data not shown). occl I and II, occludin loops I and II, respectively; clau I and II, claudin-1 loops I and II, respectively. 